\title{
One-Year Evaluation of a Targeted Medication Therapy Management Intervention for Older Adults
}

\author{
Ashley I. Martinez, PharmD, MS; Erin L. Abner, PhD; Gregory A. Jicha, MD, PhD; \\ Dorinda N. Rigsby, PharmD; Lynne C. Eckmann, PharmD; Mark J. Huffmyer, PharmD; \\ and Daniela C. Moga, MD, PhD
}

\begin{abstract}
BACKGROUND: Older adults are especially susceptible to adverse effects of inappropriate medication therapy, and anticholinergic medications are common culprits for cognitive dysfunction due to their action on the central nervous system. Medication therapy management (MTM) interventions can aid in deprescribing and reducing inappropriate medication use in older adults. However, there is sparse literature on the long-term sustainability of these interventions.
\end{abstract}

OBJECTIVES: To (a) investigate whether the deprescribing of anticholinergic medications during an 8-week randomized controlled trial (RCT) of a targeted MTM intervention is sustained at 1-year postintervention follow-up and (b) compare anticholinergic utilization trends in the study population with a large sample of similar individuals not exposed to the intervention.

METHODS: Participants in the targeted MTM (tMTM) RCT had normal cognition or mild cognitive impairment and were recruited from enrollees in a Iongitudinal study at the University of Kentucky Alzheimer's Disease Center (ADC) and thus have pertinent medical information gathered approximately annually. In this posttrial observational follow-up, sustainability of the anticholinergic deprescribing intervention was assessed in participants in the $\mathrm{RCT}$, and anticholinergic medication use trends were described from the RCT baseline (which occurred immediately following an ADC visit) to the next annual visit in all participants. Mean change in anticholinergic burden from RCT baseline to the next annual visit was estimated using analysis of covariance, and participants were compared with 2 external samples. Anticholinergic burden was measured using the Anticholinergic Drug Scale (ADS). The odds of decreasing baseline anticholinergic burden and number of total and strong anticholinergic medications at the follow-up study time point was assessed using logistic regression.

RESULTS: Of the deprescribing changes made during the initial RCT, $50 \%$ were sustained after 1 year. Participants in the tMTM trial reported decreases in the use of anticholinergic antihistamines and bladder agents ( -6.5 and $-4.4 \%$, respectively), but there was no change in the use of anticholinergic agents targeted at the central nervous system. While the anticholinergic burden of RCT participants decreased over 1 year (adjusted mean ADS change $[95 \% \mathrm{Cl}]=-0.33[-0.72,0.07])$, it was not different than the change observed in 2 external samples at the trial center $(-0.20$ $[-0.42,0.02])$ and nationally $(-0.33[-0.39,-0.26])$. There were no statistically significant differences between trial participants and external samples in the odds of decreasing anticholinergic burden nor in decreasing the number of total, or strongly anticholinergic, medications at the 1-year follow-up.

CONCLUSIONS: This study demonstrates that the sustainability of deprescribing is limited to the period of intervention, rather than affording lasting effects even over periods as short as 1 year, which was demonstrated not only in the small group of RCT participants but also by comparison with external groups. Future work should extend the duration of intervention and follow-up periods for MTM interventions to allow further insights regarding the sustainability of deprescribing efforts in older adults.

J Manag Care Spec Pharm. 2020;26(4):520-28

Copyright $\odot 2020$, Academy of Managed Care Pharmacy. All rights reserved.

\section{What is already known about this subject}

Anticholinergic medications are common culprits of adverse cognitive effects in older adults

Medication therapy management (MTM) interventions can reduce inappropriate medication use in older adults and aid in deprescribing efforts to improve health outcomes.

There is insufficient evidence regarding the sustainability of deprescribing MTM interventions over longer periods of time due to lack of studies, including sustained intervention and postintervention long-term follow-up.

\section{What this study adds}

This study demonstrates that targeted anticholinergic deprescribing during an 8-week MTM randomized controlled trial is successful, but that overall anticholinergic medication use 1 year after intervention is similar to comparable subjects nationwide. This study highlights the importance of future trials examining factors that may contribute to longer sustainability of individualized MTM interventions targeting deprescribing in older adults.

T he Medicare Prescription Drug, Improvement, and Modernization Act of 2003 formally recognized the role of medication therapy management (MTM) programs in reducing the risk of adverse drug events, ${ }^{1}$ and pharmacists play a key role in the effective implementation of MTM programs. ${ }^{2,3}$ While MTM programs can provide a range of services aimed at optimizing therapeutic outcomes and preventing costly medication-related adverse events, a core component of most MTM programs is the comprehensive medication review, ${ }^{4}$ an in-depth review of all patient medications that allows the identification of, and recommendations to address the issue of, inappropriate medication use that may contribute to cognitive impairment in the aging population. ${ }^{5}$ 
Older adults are a unique population of patients who may especially benefit from MTM interventions, since their advanced age and generally high medical comorbidity load may place them at greater risk for drug-related adverse events. ${ }^{6-8}$ The literature on MTM programs led by pharmacists has generally shown that these interventions reduce inappropriate prescribing and potential drug-related problems in the older adult population, with most studies focusing on reducing polypharmacy rather than targeting deprescribing of particular drug classes, such as anticholinergic medications that are known to be detrimental to cognitive function, ${ }^{9-13}$ yet are highly prescribed in the aging population today. ${ }^{14}$

Despite evidence of the short-term efficacy of MTM interventions, no studies have been conducted that focus primarily on the sustainability of MTM interventions in preventing potentially inappropriate medication use over longer periods of time. In a recent systematic review that analyzed approaches to improve appropriate medication therapy among those with polypharmacy, investigators included 32 studies, of which only $37.5 \%$ sustained the intervention for at least 1 year. Yet, the review did not include any reflection regarding the length of postintervention follow-up of the included MTM studies. ${ }^{11}$ There could be many factors that influence the extent to which an MTM intervention successfully sustains deprescribing efforts over time.

Previously, we reported on an 8-week, parallel-arm, randomized controlled trial (RCT) to evaluate whether a targeted patient-centered pharmacist-physician team (tMTM) intervention reduced the use of inappropriate anticholinergic medication use among older adults with normal cognition or mild cognitive impairment (MCI) enrolled in a longitudinal study at the University of Kentucky Alzheimer's Disease Center (UKADC), which is part of the National Institutes on Aging Alzheimer's Disease Centers Program. ${ }^{15}$ Standardized data collection protocols for all ADCs are coordinated by the National Alzheimer's Coordinating Center (NACC). ${ }^{16}$ The results of the initial RCT showed that the tMTM intervention resulted in reduced use of inappropriate anticholinergic medications during the 8 -week trial period. ${ }^{17}$ Unfortunately, as with most clinical trials, the short follow-up period of the RCT limited its ability to determine the sustainability of the tMTM intervention and its effect on anticholinergic medications use patterns over a longer period of time.

The present study focused on the long-term sustainability of this tMTM intervention at 1 year after intervention. ${ }^{18}$ In addition, we compared the l-year effect of an anticholinergic tMTM intervention with natural trends in similar groups of subjects not participating in the RCT from local and national comparative samples that were not exposed to the tMTM intervention.

\section{Methods}

\section{Study Design}

This report is a posttrial observational follow-up of an RCT that evaluated the effect of a tMTM intervention on deprescribing medications with anticholinergic properties among older adults with normal cognition or MCI. The tMTM intervention has been described previously. ${ }^{17}$ The RCT and this observational follow-up were approved by the University of Kentucky Institutional Review Board, and all participants provided signed informed consent for participation in research. For participants with MCI, the informed consent was also provided by their legally authorized representative. The focus of this report was on outcomes of RCT participants approximately 1 year after the trial's completion, compared with 2 external samples of similar subjects that included a local (UKADC) and a national (NACC) sample.

\section{Targeted MTM Intervention}

Participants were recruited from the UKADC and were screened for RCT eligibility between October 1, 2014, and September 30, 2015, and those potentially eligible were invited to participate in the RCT. UKADC participants were eligible if they were aged at least 65 years and reported at least 1 medication with anticholinergic properties at their annual UKADC visit. ADC participants living in a long-term care facility or with at least moderate dementia (Clinical Dementia Rating [CDR] Dementia Staging Instrument score $\geq 2$ ) were excluded. Participants were randomly assigned to either the tMTM intervention or standard of care using a simple block randomization scheme, and group allocation was not revealed until after the baseline medication review was completed by the trial pharmacists.

All participants were given generic informational materials on the importance of being actively involved with-and learning about-their medications and were encouraged to talk to their primary care providers about their medications. Participants randomized to the intervention group met with a pharmacist-clinician (MD or APRN) team with the goal of discontinuing any potentially inappropriate medications with anticholinergic properties or replacing with safer alternatives, including medication substitutions and nonpharmacologic interventions (i.e., deprescribing). When alternatives were unavailable, reduction in dosage was recommended whenever clinically indicated to reduce the anticholinergic burden. Because of ethical considerations, participants randomized to the control group received a modified version of the tMTM intervention at the end of the RCT. Specifically, these participants discussed their medications with the trial pharmacists and received recommendations regarding their anticholinergic medications. 


\begin{tabular}{|c|c|c|c|c|c|c|}
\hline & \multicolumn{2}{|c|}{$\begin{array}{c}\text { RCT Participants } \\
\mathrm{n}=46\end{array}$} & \multicolumn{2}{|c|}{$\begin{array}{l}\text { Local External Control } \\
\mathrm{n}=146\end{array}$} & $\begin{array}{l}\text { National External Control } \\
n=1,745\end{array}$ & $\begin{array}{c}P \text { Values: } \\
\text { RCT vs. Local; } \\
\text { RCT vs. National }\end{array}$ \\
\hline \multicolumn{7}{|l|}{ Baseline demographics } \\
\hline Age, mean (SD) & 77.7 & $(6.4)$ & 77.1 & $(5.8)$ & $(7.0)$ & $0.6180 ; 0.1082$ \\
\hline Female, n (\%) & & $(69.6)$ & 96 & $(65.8)$ & $1,032 \quad(59.1)$ & $0.6325 ; 0.1553$ \\
\hline White race, $\mathrm{n}(\%)$ & 43 & $(93.5)$ & 137 & $(93.8)$ & $1,443 \quad(82.7)$ & $0.9304 ; 0.0548$ \\
\hline Education years, mean (SD) & 16.3 & $(2.7)$ & 16.5 & $(2.8)$ & (3.1) & $0.7572 ; 0.2872$ \\
\hline \multicolumn{7}{|l|}{ Baseline clinical data } \\
\hline $\mathrm{CDR}>0, \mathrm{n}(\%)$ & 15 & $(32.6)$ & 48 & $(32.9)$ & $814 \quad(46.7)$ & $0.1153 ; 0.0594$ \\
\hline \multicolumn{7}{|c|}{ Number of medications, mean (SD) } \\
\hline Total & 10.5 & $(2.7)$ & 10.2 & $(3.7)$ & $(4.4)$ & $0.6784 ; 0.0474$ \\
\hline Any anticholinergic & 1.4 & $(0.7)$ & 1.3 & $(0.7)$ & $(0.7)$ & $0.5347 ; 0.7590$ \\
\hline Strong anticholinergic & 0.4 & $(0.6)$ & 0.3 & $(0.5)$ & $(0.5)$ & $0.3015 ; 0.2460$ \\
\hline ADS, mean (SD) & 2.9 & $(1.7)$ & 2.2 & $(1.6)$ & $(1.5)$ & $0.0050 ; 0.0005$ \\
\hline
\end{tabular}

\section{Posttrial Observational Follow-Up}

The main objectives of the current investigation were the following: (a) to investigate whether deprescribing anticholinergic medications during an 8-week RCT of a tMTM intervention was sustained 1 year after the intervention and (b) to compare anticholinergic utilization trends in the study population with a large sample of similar individuals not exposed to the intervention. Specifically, we compared the changes in anticholinergic medication use and anticholinergic burden from baseline (i.e., RCT enrollment) with the next UKADC visit approximately 1 year later with the natural trends in local (UKADC) and national (NACC) samples not exposed to the tMTM intervention. Sustainability was evaluated in RCT participants by describing changes in anticholinergic medication use from the end of the trial period to the next follow-up visit approximately 1 year later. We evaluated these changes in all RCT participants as a single group, since all participants received some form of the intervention whether formal (the pharmacist-clinician team tMTM intervention group) or informal (the pharmacist-led tMTM control group).

To account for historical trends in medication use, we constructed 2 external control samples of ADC participants who were not enrolled in the RCT and examined their anticholinergic medication changes over the same period of time. These external control samples were identified from the database of participants enrolled in the UKADC, as well as all current and former ADCs throughout the United States. The National Institute on Aging (NIA) began the ADC program in 1984 as a comprehensive effort to boost research on Alzheimer disease and related disorders. ${ }^{15}$ ADCs prospectively collect the Uniform Data Set (UDS), which includes standardized demographic, clinical, neuropsychological, and diagnostic patient data, and share deidentified UDS data with the NACC. ${ }^{19}$ Annual ADC visit data were then used to assess changes in medication use after 1 year; visits were selected such that the first visit occurred in either 2014 or 2015 (similar to the timeline of the original RCT), and subjects were selected by applying the RCT inclusion and exclusion criteria to all subjects. ${ }^{17}$ Participants who did not have medication information available at the annual visit after baseline were excluded from this analysis. Thus, all participants had information on medication use at baseline and the next annual ADC visit.

The first external ADC control group included local subjects from the UKADC who met inclusion and exclusion criteria in 2014-2015 but were not enrolled in the RCT. Because all RCT participants were recruited from the UKADC, this group was intended to provide information about anticholinergic medication use trends for the source population of similar older adults residing in the same geographical area as the RCT participants. The second external control group included all eligible subjects from the other ADCs (excluding UKADC), based on UDS data obtained from the NACC. Because the UKADC is part of the national ADC program, this second ADC control group was included with the intent of providing information about patterns of anticholinergic medication use in a larger population with similar characteristics to the RCT participants but who resided in geographically disparate locations.

\section{Outcome Measures}

This posttrial observational follow-up focused on 3 outcome measures to describe anticholinergic medication use: (1) patterns of anticholinergic medications use, (2) mean change in anticholinergic burden, and (3) odds of decreasing use of anticholinergic medications. For participants in the RCT, we investigated patterns of anticholinergic medications use from the end of the trial period to the next ADC visit approximately 1 year later, with medications organized by therapeutic class. 


\begin{tabular}{|c|c|c|}
\hline TABLE 2 & $\begin{array}{l}\text { prescribed Anti } \\
\text { dication Sustai } \\
\text { T Participants }\end{array}$ & $\begin{array}{l}\text { holinergic } \\
\text { bility Among }\end{array}$ \\
\hline Class & Medication & 1-Year Outcome \\
\hline \multirow{6}{*}{ Antidepressants } & \multirow{2}{*}{ Amitriptyline } & Reinitiated \\
\hline & & Reinitiated \\
\hline & Citalopram & Discontinued \\
\hline & \multirow{2}{*}{ Fluoxetine } & Discontinued \\
\hline & & Replaced with citalopram \\
\hline & Paroxetine & Reinitiated \\
\hline \multirow{5}{*}{ Antihistamine } & \multirow{2}{*}{ Diphenhydramine } & Discontinued \\
\hline & & Reinitiated \\
\hline & \multirow{3}{*}{ Loratadine } & Discontinued \\
\hline & & Discontinued \\
\hline & & Reinitiated \\
\hline \multirow{3}{*}{ Bladder } & Oxybutynin & Discontinued \\
\hline & \multirow{2}{*}{ Tolterodine } & Reinitiated \\
\hline & & Reinitiated \\
\hline Cardiovascular & Isosorbide & Discontinued \\
\hline \multirow{2}{*}{$\begin{array}{l}\text { Central nervous } \\
\text { system-active }\end{array}$} & Clonazepam & Discontinued \\
\hline & Tramadol & Discontinued \\
\hline \multirow{5}{*}{ Gastrointestinal } & Cimetidine & Discontinued \\
\hline & Dicyclomine & Reinitiated \\
\hline & \multirow{3}{*}{ Ranitidine } & Reinitiated \\
\hline & & Reinitiated \\
\hline & & Discontinued \\
\hline
\end{tabular}

We evaluated the mean change in anticholinergic burden and the odds of decreasing use of anticholinergic medications from the baseline to next ADC visit (approximately 1 year later) between all RCT participants and the 2 external control groups. "Decreased" use of anticholinergic medication was defined as report of fewer medications with any anticholinergic properties at the next ADC visit than at baseline.

Anticholinergic burden was measured using the updated version of the Anticholinergic Drug Scale (ADS), ${ }^{20}$ which accurately quantifies anticholinergic burden and categorizes the largest number of medications for anticholinergic activity of all currently available scales. ${ }^{21,22}$ The ADS has 4 levels for each included drug, ranging from 0 (no known anticholinergic activity) to 3 (marked anticholinergic activity, or strongly anticholinergic). The summation of anticholinergic activity level for all reported medications reflects the total anticholinergic burden for the participant (higher scores indicate a higher burden).

Odds of decreased use of any or only strongly (defined as ADS =3) anticholinergic medications over the follow-up period were also considered. These outcomes were included to provide more clinically relevant results, since a count of medications may be more easily measured and implemented in clinical practice than changes in anticholinergic burden, and strongly anticholinergic medications may be more easily prioritized for deprescribing.

\section{Statistical Analysis}

We used the Student's t-test or Wilcoxon rank sum test for continuous variables and the Pearson chi-square test or Fisher's exact test for binary variables to assess the similarity of RCT participants and external control subjects at their respective baseline visits.

Using a table organized by medication class, we listed deprescribed anticholinergic medications among RCT participants and described whether these medications remained discontinued, were replaced, or were reinitiated by the 1-year postintervention follow-up. Mean ADS change was assessed using analysis of covariance (ANCOVA), controlling for imbalanced baseline covariates (total number of medications and baseline ADS) to generate adjusted means and 95\% confidence intervals (CIs). The ANCOVA aimed to compare mean ADS changes of RCT participants with local and national external groups to determine whether participation in the RCT was associated with greater reduction in anticholinergic burden than standard of care. To determine whether there was a difference in the odds of decreasing anticholinergic burden, total number, or number of strong anticholinergic medications, we used logistic regression to compare groups with adjusted odds ratios (ORs) in the same fashion as the ANCOVAs, controlling for total number of anticholinergic medications and ADS at baseline. All statistical analyses were conducted in SAS version 9.4 (SAS Institute, Cary, NC).

\section{Results}

\section{Baseline Characteristics}

In total, 1,937 ADC participants were included in analyses ( $n=46,146$, and 1,745 from the RCT, local, and national groups, respectively). Groups were similar in demographic characteristics, including age (mean 76.9 years), female sex (64.8\%), white race (83.8\%), and years of education (mean 15.9 years; Table 1). There was a similar proportion of participants with impaired cognition in each group, but RCT participants reported more medications in general at baseline than those in the NACC group $(P=0.048)$ and had a higher anticholinergic burden than the UKADC and NACC groups $(P=0.005$ and 0.001 , respectively).

\section{Anticholinergic Medication Use Patterns}

At baseline, the most commonly used anticholinergic medication class in all groups was antidepressants $(32.6 \%, 41.1 \%$, and $46.0 \%$ of participants in the RCT, UKADC, and NACC groups, respectively). Bladder antimuscarinics and anticholinergic cardiovascular agents were also highly used.

Among the 46 participants who had postintervention follow-up data, there were 77 anticholinergic medications used at baseline. The most commonly used anticholinergic medication classes included antidepressants $(n=18,23.4 \%)$, cardiovascular medications $(n=15,19.5 \%)$, and bladder agents $(n=14,18.2 \%)$. 


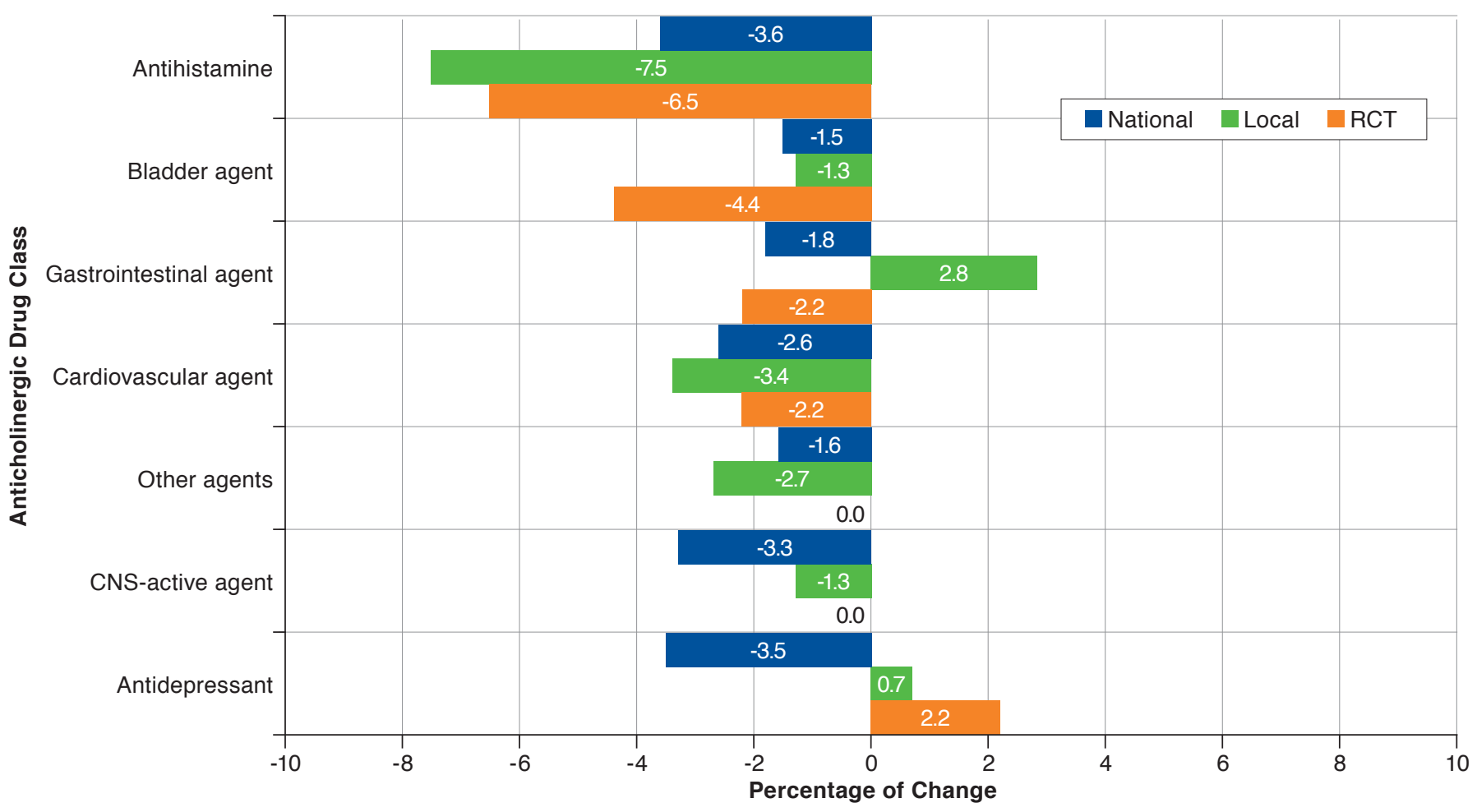

CNS = central nervous system; $R C T=$ randomized controlled trial.

Of the anticholinergic medications reported at baseline, $22(28.6 \%)$ were discontinued at the end of the 8-week RCT (27.3\% antidepressants, $22.8 \%$ each of antihistamines and gastrointestinal agents, $13.6 \%$ bladder agents, $9.1 \%$ central nervous system [CNS]-targeted agents excluding antidepressants, and $4.5 \%$ cardiovascular agents; Table 2). All of the deprescribed CNS-targeted and cardiovascular agents remained discontinued at the 1-year postintervention follow-up, while $60 \%, 40 \%, 33 \%$, and $33 \%$ of the antihistamines, gastrointestinal agents, bladder agents, and antidepressants remained discontinued, respectively. Overall, $50 \%$ of the anticholinergic medications that were deprescribed remained discontinued at the 1-year postintervention period, although success varied by anticholinergic medication class.

Of the baseline anticholinergic medications, 55 (71.4\%) were continued at the end of the 8-week RCT, but 16 of these (29.1\%) were discontinued by the 1-year postintervention follow-up. These included antihistamines ( $\mathrm{n}=5,31.75 \%)$; bladder and cardiovascular agents ( $\mathrm{n}=4$ and $\mathrm{n}=3,25 \%$ and $18.75 \%$ respectively); and one each of antidepressant, gastrointestinal, CNS-targeted, and steroid agents.
The difference in the percentage of participants using each anticholinergic medication between baseline and the next follow-up visit approximately 1 year later (by class) is visualized in Figure 1. RCT participants reported the largest decreases in antihistamines ( $-6.5 \%$ difference between baseline and 1-year use), followed by bladder antimuscarinics $(-4.4 \%)$. The 2 external control samples did not report as large of a decrease in the use of bladder antimuscarinics. However, while both external control samples saw marked decreases in the use of CNS-active anticholinergic medications, there was no change between baseline and 1 year in the frequency of RCT participants using such medications. After 1 year, a slightly greater proportion of RCT participants reported using anticholinergic antidepressants $(+2.2 \%)$ compared with these same subjects at baseline.

\section{Change in Anticholinergic Burden and Medication Use}

After adjusting for baseline total number of medications and baseline anticholinergic burden, RCT participants had an average 0.33-point decrease in ADS from baseline to 1 year (95\% $\mathrm{CI}=-0.72,0.07$ ). As seen in Table 3 , this was not statistically significantly different than the change in mean ADS seen in either local or national external control samples (mean [95\% 


\begin{tabular}{|c|c|c|c|}
\hline TABLE 3 & \multicolumn{3}{|c|}{$\begin{array}{l}\text { Changes in Anticholinergic } \\
\text { Medication Use }\end{array}$} \\
\hline & $\begin{array}{c}\text { RCT } \\
\text { Participants } \\
\mathbf{n}=46\end{array}$ & $\begin{array}{c}\text { Local External } \\
\text { Control } \\
\mathbf{n}=146\end{array}$ & $\begin{array}{c}\begin{array}{c}\text { National } \\
\text { External Control } \\
\mathrm{n}=1,745\end{array} \\
\end{array}$ \\
\hline $\begin{array}{l}\text { ADS change, mean } \\
(95 \% \mathrm{CI})^{\mathrm{a}}\end{array}$ & $\begin{array}{c}-0.33 \\
(-0.72,0.07)^{\mathrm{b}}\end{array}$ & $\begin{array}{c}-0.20 \\
(-0.42,0.02) \\
\end{array}$ & $\begin{array}{c}-0.33 \\
(-0.39,-0.26)\end{array}$ \\
\hline \multicolumn{4}{|l|}{ Decreased, n (\%) } \\
\hline ADS & $16 \quad(34.8)$ & $40 \quad(27.4)$ & $508 \quad(29.2)$ \\
\hline $\begin{array}{l}\text { Number of } \\
\text { anticholinergics }\end{array}$ & $13 \quad(28.3)$ & $37 \quad(25.3)$ & $486 \quad(27.9)$ \\
\hline $\begin{array}{l}\text { Number of strong } \\
\text { anticholinergics }\end{array}$ & $(8.7)$ & $(9.6)$ & (9.8) \\
\hline \multicolumn{4}{|c|}{$\begin{array}{l}\text { adjusted for baseline number of medications and anticholinergic burden. } \\
\text { bNot statistically significantly different from local or national samples; } P=0.58 \text { and } \\
0.28, \text { respectively. } \\
\text { ADS=Anticholinergic Drug Scale; } C I=\text { confidence interval; } R C T=\text { randomized } \\
\text { controlled trial. }\end{array}$} \\
\hline
\end{tabular}

CI] $-0.20[-0.42,0.02]$ and $-0.33[-0.39,-0.26]$ for the local and national groups, respectively).

A greater proportion of RCT participants decreased anticholinergic burden (34.8\% among RCT participants vs. $27.4 \%$ and $29.2 \%$ in the local and national groups; Table 3) and the number of anticholinergic medications (28.3 vs. 25.3 and 27.9\%) compared with the external control groups. However, this difference was not statistically significant, since RCT participants had similar odds of decreased anticholinergic burden (OR $[95 \% \mathrm{CI}]=1.07[0.51,2.25]$ and $0.95[0.50,1.80])$ and decreased number of anticholinergic medications (OR [95\% $\mathrm{CI}]=0.92[0.43,1.98]$ and $0.78[0.40,1.53])$ compared with the local and national external controls, respectively (Figure 2).

When restricted to strongly anticholinergic medications (i.e., $\mathrm{ADS} \geq 3$ ), $8.7 \%$ of RCT participants decreased the use of these medications after 1 year, compared with $9.6 \%$ and $9.8 \%$ of those in the local and national external groups. There was no difference in the odds of decreasing number of strongly anticholinergic medications among RCT participants when compared with the local and national control groups (OR [95\% $\mathrm{CI}]=0.55[0.15,1.98]$ and $0.50[0.16,1.55])$.

\section{Discussion}

Existing literature on deprescribing medications in older adults indicates that these interventions improve appropriate medication use and health outcomes. ${ }^{11,23}$ However, few studies follow subjects for extended periods of time, and even fewer measure medication use more than once. As a result, there is a paucity of data on the effectiveness and sustainability data for deprescribing MTM interventions in the literature.

The present study evaluates the 1-year sustainability of a short-term deprescribing tMTM intervention to reduce inappropriate anticholinergic medications. We found that the marked decrease in anticholinergic burden seen in the formal intervention group during the RCT was attenuated after approximately 1 year (mean ADS change $[\mathrm{SE}]=-1.0$ [0.3] after 8-weeks compared with -0.33 [0.2] after approximately 1 year). ${ }^{17}$ While the short-term findings from the RCT are in line with existing evidence, the decreased effect size over time found in this follow-up was more pronounced than in other longer-term deprescribing studies. ${ }^{11}$ Although there are only a limited number of pharmacist-led deprescribing studies in community-dwelling older adults that have followed subjects for at least 1 year, most describe a more significant reduction in measures of inappropriate medication use than was found in this study. ${ }^{24-27}$ One potential explanation for this discrepancy is that this is the first deprescribing intervention that uses an MTM strategy targeting a specific medication class instead of the entire medication regimen.

Indeed, even within this study we showed that the degree of sustainability varied by anticholinergic medication indication (all CNS-acting and cardiovascular agents remained discontinued, while only 33\% of antidepressants and bladder agent discontinuations were sustained). Notably, there are many nonanticholinergic medications available to treat CNS and cardiovascular conditions, while nearly all agents used to treat overactive bladder are anticholinergic. Alternative agents are a key component to ensuring that patient preferences are taken into consideration, since without an effective alternative patients may reinitiate. ${ }^{28,29}$ Thus, the attenuated sustainability of this tMTM intervention may have been related to its targeted nature, which limited the pool of available alternatives. In line with current literature,$^{11}$ these findings suggest that balancing patient-specific goals with reducing anticholinergic burden is crucial to longterm sustainability for deprescribing interventions.

In this posttrial observational follow-up, we also compared RCT participants (who were recruited from the UKADC) with 2 different external groups: (1) participants in the UKADC who did not participate in the RCT and (2) participants enrolled in ADCs throughout the country. All groups experienced a small, clinically insignificant reduction in the use of anticholinergic medications over the approximately 1-year follow-up. In addition, RCT participants had similar odds of decreasing the number of total and strong anticholinergic medications used after 1 year as the external control samples. Although we noted that all these groups decreased with regard to anticholinergic use, we cannot clearly determine the reason behind this change. It is likely that external factors influencing care for older adults played a role in our observed trends, including an increased awareness in the potential harms caused by anticholinergic medications.

In addition, this finding may be related to the fact that the average number of anticholinergic medications used at baseline was less than 2, leaving little room for improvement. This stands in contrast to most community deprescribing 


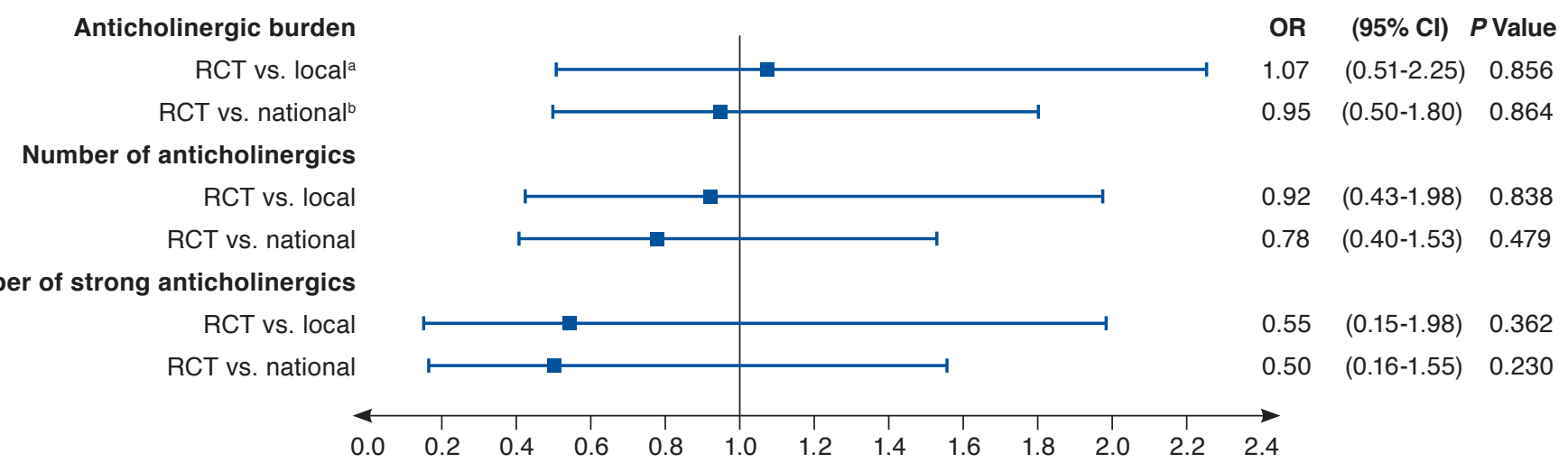

a RCT vs. local: randomized controlled trial participants versus local (University of Kentucky) Alzheimer's Disease Center participants.

${ }^{b} R C T$ vs. national: randomized controlled trial participants versus national Alzheimer's Disease Center participants.

$C I=$ confidence intervals; $O R=$ odds ratio; $R C T=$ randomized controlled trial.

interventions that focus on subjects with polypharmacy, where the baseline number of medications is high. The external control groups-a sample of individuals from the local ADC that served as the source population for the RCT and ADC participants from around the country-served as an important contextualization, providing a picture of what trajectory RCT participants might have taken had they not participated in the trial. This comparison highlights the need to further examine anticholinergic medication use in this population and determine what steps can be taken to further reduce the use of this dangerous medication class. ${ }^{30-32}$

Given that patients are generally interested in reducing inappropriate medication use and that they value communication and interaction with their health care providers in the medication decision-making process, ${ }^{33,34}$ future deprescribing interventions should provide patient-specific recommendations and strive to engage patients throughout the process, including after initial deprescribing, to ensure sustainability. Pharmacists provide an accessible and important health care resource who may be able to maintain continual patient relationships through the MTM and deprescribing process. In support of this notion, a recent pilot study showed that patients may be more willing to follow-up with pharmacists when they work in a team with primary care providers. ${ }^{35} \mathrm{~A}$ larger RCT with a follow-up of 12 months and repeated patient interaction to address long-term sustainability is underway. ${ }^{36}$ Future studies of MTM deprescribing interventions should include longer-term follow-up of participants to provide more data on the characteristics of interventions that sustain over longer periods of time.

\section{Limitations}

This study has some limitations to consider. RCT participants and both external control samples were selected from ADCs and were recruited into these centers via clinician or community organization referral or were volunteers interested in contributing to dementia research. Thus, RCT participants and ADC participants in the external control groups were highly educated and cannot be considered a nationally representative sample of older adults, which limits the generalizability of our results. In addition, medication use for participants in the study was based on self-report through a brown-bag medication review approach, except for the baseline and visits at the end of the trial period for the trial participants.

Another key caveat to interpreting these findings lies in the lack of a universally agreed-on rating scale for anticholinergic burden. It is possible that another measurement tool for anticholinergic burden would have provided different results.

Also, this current investigation was observational. Generally, observational studies can make only weak causal inferences based on results due to less control for confounding factors. However, the adjustment for imbalanced baseline covariates and addition of the external control groups increases this study's strength to make causal inferences about the sustainability of the tMTM deprescribing intervention.

Finally, given the sample size of the RCT, we were underpowered to detect small changes in ADS over the 1-year follow-up. Nevertheless, the effect size of the RCT estimates were similar to those in the external control groups, leading us to the conclusion that there was little clinically meaningful sustainability. Thus, even beyond the interpretation of the numbers, our study shed light into important aspects of medication optimization and deprescribing interventions, including sustainability. 


\section{Conclusions}

Pharmacist-engaged, multidisciplinary MTM programs are important tools for deprescribing and optimizing medication-related outcomes in older adult patients. However, in this posttrial observational follow-up, we did not find significant differences in the sustainability of reductions in anticholinergic burden or in number of anticholinergic or strongly anticholinergic medications used between participants in a tMTM intervention and similar groups of community-dwelling older adults at a 1-year follow-up. Future studies should explore how optimizing patient engagement and providing ongoing deprescribing support may improve sustainability of MTM interventions. Studies also should evaluate interventions to enhance and optimize communication between patients, pharmacists, and primary care providers to facilitate and sustain deprescribing.

\section{Authors}

ASHLEY I. MARTINEZ, PharmD, MS, Department of Pharmacy Practice and Science, College of Pharmacy, University of Kentucky, Lexington. ERIN L. ABNER, PhD, Department of Epidemiology, College of Public Health, University of Kentucky, and SandersBrown Center on Aging, Lexington, Kentucky. GREGORY A. JICHA, $\mathrm{MD}, \mathrm{PhD}$, Sanders-Brown Center on Aging, Lexington, Kentucky, and Department of Neurology, College of Medicine, University of Kentucky, Lexington. DORINDA N. RIGSBY, PharmD, VA Medical Center, Lexington, Kentucky. LYNNE C. ECKMANN, PharmD, PRO2RX LLC Pharmacy Consulting Services, Lexington, Kentucky. MARK J. HUFFMYER, PharmD, Department of Pharmacy Practice and Science, College of Pharmacy, University of Kentucky, and PRO2RX LLC Pharmacy Consulting Services, Lexington, Kentucky. DANIELA C. MOGA, MD, PhD, Department of Pharmacy Practice and Science, College of Pharmacy, and Department of Epidemiology, College of Public Health, University of Kentucky, and Sanders-Brown Center on Aging, Lexington, Kentucky.

AUTHOR CORRESPONDENCE: Daniela C. Moga, MD, PhD, Department of Pharmacy Practice and Science, College of Pharmacy, University of Kentucky, 789 S. Limestone, Lee T. Todd Jr. Bldg., Rm 241, Lexingon, KY 40536. Tel.: 859.323.9682;

E-mail: daniela.moga@uky.edu.

\section{DISCLOSURES}

The original trial was supported by a pilot study award from the University of Kentucky Center for Clinical and Translational Sciences (UL1TR000117). Additional support for this study was provided by the National Institutes of Health/National Institute on Aging (R01 AG054130). Jicha reports contract research for Esai, Biohaven, Alltech, Suven, Novartis, and Lilly. The other authors have nothing to disclose.

\section{ACKNOWLEDGMENTS}

The authors acknowledge Dr. Richard R. Murphy and Beth B. Coy for their contributions to the original trial, as well as Dr. Patricia Freeman for providing feedback on the earlier versions of the manuscript.

The National Alzheimer's Coordinating Center (NACC) database is funded by National Institute on Aging (NIA)/National Institutes of Health (NIH) Grant U01 AG016976. NACC data are contributed by the NIA-funded Alzheimer's Disease Centers (ADCs): P30 AG019610 (PI Eric Reiman, MD), P30 AG013846 (PI Neil Kowall, MD), P50 AG008702 (PI Scott Small, MD), P50 AG025688 (PI Allan Levey, MD, PhD), P50 AG047266 (PI Todd Golde, MD, PhD), P30 AG010133 (PI Andrew Saykin, PsyD), P50 AG005146 (PI Marilyn Albert, PhD), P50 AG005134 (PI Bradley Hyman, MD, PhD), P50 AG016574 (PI Ronald Petersen, MD, PhD), P50 AG005138 (PI Mary Sano, PhD), P30 AG008051 (PI Thomas Wisniewski, MD), P30 AG013854 (PI M Marsel Mesulam, MD), P30 AG008017 (PI Jeffrey Kaye, MD), P30 AG010161 (PI David Bennett, MD), P50 AG047366 (PI Victor Henderson, MD, MS) P30 AG010129 (PI Charles DeCarli, MD), P50 AG016573 (PI Frank LaFerla, PhD), P50 AG005131 (PI James Brewer, MD, PhD), P50 AG023501 (PI Bruce Miller, MD), P30 AG035982 (PI Russell Swerdlow, MD), P30 AG028383 (PI Linda Van Eldik, PhD), P30 AG053760 (PI Henry Paulson, MD, PhD), P30 AG010124 (PI John Trojanowski, MD, PhD), P50 AG005133 (PI Oscar Lopez, MD), P50 AG005142 (PI Helena Chui, MD), P30 AG012300 (PI Roger Rosenberg, MD), P30 AG049638 (PI Suzanne Craft, PhD), P50 AG005136 (PI Thomas Grabowski, MD), P50 AG033514 (PI Sanjay Asthana, MD, FRCP), P50 AG005681 (PI John Morris, MD), P50 AG047270 (PI Stephen Strittmatter, $\mathrm{MD}, \mathrm{PhD}$ )

\section{REFERENCES}

1. Siegel S. An introduction to the Medicare Prescription Drug, Improvement, and Modernization Act of 2003. Health Care Law Mon. 2004;Jan:3-14.

2. Smith M, Bates DW, Bodenheimer T, Cleary PD. Why pharmacists belong in the medical home. Health Aff (Millwood). 2010;29(5):906-13.

3. Maracle HL, de Oliveira DR, Brummel A. Primary care providers' experiences with pharmaceutical care-based medication therapy management services. Innov Pharm. 2012;3(1, Article 72). Available at: https://pubs.lib.umn. edu/index.php/innovations/article/view/255. Accessed March 1, 2020

4. American Pharmacists Association, National Association of Chain Drug Stores Foundation. Medication therapy management in pharmacy practice: core elements of an MTM service model (version 2.0). J Am Pharm Assoc (2003). 2008;48(3):341-53.

5. Bluml BM. Definition of medication therapy management: development of professionwide consensus. J Am Pharm Assoc (2003). 2005;45(5):566-72.

6. Lau DT, Kasper JD, Potter DEB, Lyles A, Bennett RG. Hospitalization and death associated with potentially inappropriate medication prescriptions among elderly nursing home residents. Arch Intern Med. 2005;165(1):68-74.

7. Farrell B, Szeto W, Shamji S. Drug-related problems in the frail elderly. Can Fam Physician. 2011;57(2):168-69.

8. Gu Q, Paulose-Ram R, Burt V, Kit B. Prescription cholesterol-lowering medication use in adults aged 40 and over: United States, 2003-2012. NCHS data brief, no. 177. National Center for Health Statistics. 2014. Available at: http:// www.cdc.gov/nchs/data/databriefs/db177.pdf. Accessed March 1, 2020.

9. Kallio SE, Kiiski A, Airaksinen MSA, et al. Community pharmacists' contribution to medication reviews for older adults: a systematic review. J Am Geriatr Soc. 2018;66(8):1613-20.

10. Page AT, Clifford RM, Potter K, Schwartz D, Etherton-Beer CD. The feasibility and effect of deprescribing in older adults on mortality and health: a systematic review and meta-analysis. Br J Clin Pharmacol. 2016;82(3):583-23.

11. Rankin A, Cadogan CA, Patterson SM, et al. Interventions to improve the appropriate use of polypharmacy for older people. Cochrane Database Syst Rev. 2018 Sep 3;9:CD008165. 
12. 2019 American Geriatrics Society Beers Criteria Update Expert Panel. American Geriatrics Society 2019 Updated AGS Beers Criteria for Potentially Inappropriate Medication Use in Older Adults. J Am Geriatr Soc. 2019;67(4):674-94.

13. Ancelin ML, Artero S, Portet F, Dupuy A-M, Touchon J, Ritchie K. Nondegenerative mild cognitive impairment in elderly people and use of anticholinergic drugs: longitudinal cohort study. BMJ. 2006;332(7539):455-59.

14. Kachru N, Carnahan RM, Johnson ML, Aparasu RR. Potentially inappropriate anticholinergic medication use in older adults with dementia. J Am Pharm Assoc (2003). 2015;55(6):603-612.

15. National Institute on Aging. Alzheimer's Disease Research Centers. 2018. Bethesda, MD. Available at: https://grants.nih.gov/grants/guide/rfafiles/RFA-AG-20-004.html. Accessed March 1, 2020.

16. Morris JC, Weintraub S, Chui HC, et al. The Uniform Data Set (UDS): clinical and cognitive variables and descriptive data from Alzheimer Disease Centers. Alzheimer Dis Assoc Disord. 2006;20(4):210-216.

17. Moga DC, Abner EL, Rigsby DN, et al. Optimizing medication appropriateness in older adults: a randomized clinical interventional trial to decrease anticholinergic burden. Alzheimers Res Ther. 2017;9(1):36.

18. Llewellyn-Bennett R, Bowman L, Bulbulia R. Post-trial follow-up methodology in large randomized controlled trials: a systematic review protocol. Syst Rev. 2016;5(1):214.

19. Besser L, Kukull W, Knopman DS, et al. Version 3 of the National Alzheimer's Coordinating Center's Uniform Data Set. Alzheimer Dis Assoc Disord. 2018;32(4):351-58

20. Carnahan RM, Lund BC, Perry PJ, Pollock BG, Culp KR. The Anticholinergic Drug Scale as a measure of drug-related anticholinergic burden: associations with serum anticholinergic activity. J Clin Pharmacol. 2006:46(12):1481-86.

21. Salahudeen MS, Duffull SB, Nishtala PS. Anticholinergic burden quantified by anticholinergic risk scales and adverse outcomes in older people: a systematic review. BMC Geriatr. 2015;15:31.

22. Lozano-Ortega G, Johnston KM, Cheung A, et al. A review of published anticholinergic scales and measures and their applicability in database analyses. Arch Gerontol Geriatr. 2020;87:103885. Available at: https://www. sciencedirect.com/science/article/pii/S0167494319301207?via\%3Dihub. Accessed March 8, 2020

23. Viswanathan M, Kahwati LC, Golin CE, et al. Medication therapy management interventions in outpatient settings. Report no.: 14(15)-EHC037-EF Agency for Healthcare Research and Quality. 2014. Available at: http://www. ncbi.nlm.nih.gov/books/NBK294489/. Accessed March 1, 2020.
24. Campins L, Serra-Prat M, Gózalo I, et al. Randomized controlled trial of an intervention to improve drug appropriateness in community-dwelling polymedicated elderly people. Fam Pract. 2017;34(1):36-42.

25. Hanlon JT, Weinberger M, Samsa GP, et al. A randomized, controlled trial of a clinical pharmacist intervention to improve inappropriate prescribing in elderly outpatients with polypharmacy. Am J Med. 1996;100(4):428-37. 26. Olsson IN, Runnamo R, Engfeldt P. Drug treatment in the elderly: an intervention in primary care to enhance prescription quality and quality of life. Scand J Prim Health Care. 2012;30(1):3-9.

27. Taylor CT, Byrd DC, Krueger K. Improving primary care in rural Alabama with a pharmacy initiative. Am J Health Syst Pharm. 2003;60(11):1123-29.

28. World Health Organization. Patient Engagement. Geneva: World Health Organization; 2016. Available at: https://apps.who.int/iris/bitstream/hand le/10665/252269/9789241511629-eng.pdf;jsessionid=2B5ECAC91E8802DB2 C20595A7E3EA0B7?sequence $=1$. Accessed March 1, 2020

29. American Pharmacists Association. Pharmacists role in promoting medication adherence. In: Report of the 2015 APhA House of Delegates. J Am Pharm Assoc. 2015;55(4):365.

30. Boustani M, Campbell N, Munger S, Maidment I, Fox C. Impact of anticholinergics on the aging brain: a review and practical application. Aging Health. 2008;4(3):311-20

31. Lampela P, Lavikainen P, Garcia-Horsman JA, Bell JS, Huupponen R, Hartikainen S. Anticholinergic drug use, serum anticholinergic activity, and adverse drug events among older people: a population-based study. Drugs Aging. 2013;30(5):321-30

32. Rudolph JL, Salow MJ, Angelini MC, McGlinchey RE. The anticholinergic risk scale and anticholinergic adverse effects in older persons. Arch Intern Med. 2008;168(5):508-13.

33. Holmes HM, Todd A. The role of patient preferences in deprescribing. Clin Geriatr Med. 2017;33(2):165-75.

34. Martin P, Tannenbaum C. A realist evaluation of patients' decisions to deprescribe in the EMPOWER trial. BMJ Open. 2017;7(4):e015959.

35. AHRQ Health Care Innovations Exchange. Implementation of a medication therapy management intervention in ambulatory care settings: experiences and lessons learned from the MyRx Pilot. 2016. Available at: https:// innovations.ahrq.gov/qualitytools/implementation-medication-therapy-management-intervention-ambulatory-care-settings. Accessed March 1, 2020.

36. Moga DC, Jicha GJ. The INCREASE Study-Delaying the onset of Alzheimer's symptomatic expression. Clinical Trials.gov Identifier: NCT02849639. 2016. Available at: https://clinicaltrials.gov/ct2/show/ NCT02849639. Accessed March 1, 2020. 